\title{
HACIA UN ENCUENTRO ENTRE ANTROPOLOGÍA Y COMUNICACIÓN EN EL ANÁLISIS DE PRODUCCIONES MEDIÁTICAS

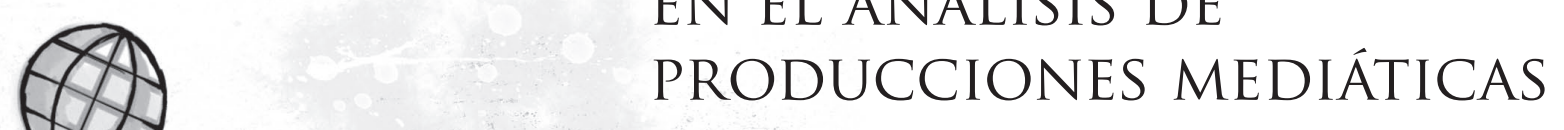

\author{
TOWARDS AN ENCOUNTER \\ BETWEEN ANTHROPOLOGY AND \\ COMMUNICATION IN THE ANALYSIS OF \\ MASS MEDIA PRODUCTIONS
}

EM DIREÇÃO AO ENCONTRO DA ANTROPOLOGIA E A COMUNICAÇÃO NA ANÁLISE DE PRODUÇÕES DA MÍDIA

Por

\section{Juliana Echeverry Ruano}

Maestra en Antropología Visual Facultad Latinoamericana de Ciencia Sociales julianaecheverry23@gmail.com Ecuador

Resumen: El presente artículo, como su nombre lo indica, plantea la necesidad de un encuentro entre antropología y comunicación en el análisis de producciones mediáticas. Su intención es cuestionar la idea de que es posible estudiar los medios de comunicación como entidades autónomas, cognoscentes y sujetas a una única racionalidad, para proponer la existencia de una relación dialéctica entre medios, producción y recepción. El texto aborda algunos de los enfoques clásicos y contemporáneos en los estudios sobre medios, e inserta en la discusión conceptos provenientes de la antropología que complementan teórica y metodológicamente esta línea investigativa tan relevante para ambos campos del conocimiento (la comunicación y la antropología).

Palabras clave: Medios de comunicación, Antropología, Cultura, Recepción y Producción 


\begin{abstract}
This paper, as its title shows, proposes the necessity of an encounter between anthropology and communication in the analysis of mass media productions. Its intention is to dispute the idea that claims it is possible to study the media as autonomous and cognizant entities that are subject to only one type of rationality. The article proposes the existence of a dialectic relationship between media, production and reception. This text addresses some classic and contemporary approaches in research on media, and introduces into the discussion some concepts from anthropology that theoretically and methodologically complement this line of research that is relevant for both spheres of knowledge (communication and anthropology).
\end{abstract}

Keywords: Media, anthropology, culture, reception and production.

Resumo: Este artículo manifesta a necessidade de um encontro da antropologia e a comunicação na análise de produções da mídia. A sua intenção é questionar a ideia de que é possível estudar a mídia como entidades autônomas, cognoscentes e sujeitas de só um tipo de racionalidade. Propõe a existência de uma relação dialética entre a mídia, produção e recepção. O texto trata alguns dos enfoques clássicos e contemporâneos nos estudos da mídia e introduz na discussão conceitos da antropologia que complementam de maneira teórica e metodológica esta lina de pesquisa tão relevante para os dois campos do conhecimento (a comunicação e a antropologia).

Palavras-chave: Mídia, antropologia, cultura, recepção e produção.

Este artículo pondrá en diálogo algunos de los enunciados teóricos que plantean la relación entre poder y medios de comunicación, para después complementarlos con ideas provenientes de la antropología. La intención es proponer un encuentro entre antropología y comunicación que sea útil para complejizar algunas ideas respecto a la producción y el consumo de los productos mediáticos.

\title{
Los medios de comunicación
}

Los medios masivos de comunicación han sido, desde su aparición, elementos centrales en la sociedad y por esta razón ha habido extensos y complejos debates sobre su condición, su poder y sus lógicas. Uno de los autores que ha delineado la historia de estas discusiones es James Curran en su libro "Medios de comunicación y poder en una sociedad democrática”. Para empezar este texto, serán mencionadas tres de los enfoques que él cita: el funcionalismo radical, la escuela de Birmingham y el liberalismo pluralista. 
James Curran (2005) afirma que durante las décadas de 1970 y 1980 se desarrolló una teoría acerca de los medios que intentó eclipsar la tradición dominante de los estudios norteamericanos de comunicación y se convirtió, por un periodo corto de tiempo, en una nueva ortodoxia. Este enfoque europeo planteaba tres características de los medios de comunicación:

(1) Se controlan por medio de la censura del mercado, la influencia del Estado y la ideología del capitalismo, (2) representan el mundo de formas selectivas que respaldan el orden social (y 3) constituyen potentes agencias de persuasión que activamente generan consentimiento con el sistema social y que resultan centrales para su mantenimiento (Curran, 2005, p. 121).

Como lo señala Curran, la afirmación de que los medios de comunicación interpretan el mundo de formas que apoyan el orden dominante aparece expuesta en incontables publicaciones (Elliot, 1980, Milibanda, 1973, Downing, 1980). Según esta perspectiva radical, los medios de comunicación “engañan al público con su representación errónea de la verdadera naturaleza y la verdadera dinámica del poder” (Curran, 2005, p. 122). Esta tradición se instaló sobre todo en Europa, pero también encontró apoyo en otros lugares, teniendo como sus exponentes más notables a Edward Herman y Noam Chomsky.

La comprensión de los medios de comunicación de los funcionalistas radicales estaba sustentada en una idea de la sociedad como lugar de dominación. Sin embargo, fue esta misma visión de sociedad la que terminó por desvirtuar su teoría. Esta visión de las personas como ilusos pre-condicionados fue cuestionada principalmente por el Centro de Estudios Contemporáneos de la Universidad de Birmingham, quienes estaban bastante entusiasmados con el estudio de algunas subculturas urbanas, cuyas acciones calificaban como rituales de resistencia que ponían en juego definiciones culturales contrarias a las dominantes. El hecho de que estas subculturas existieran, era para los teóricos de Birmingham la muestra inequívoca de que no existía una única racionalidad imposible de subvertir. Esto estimuló en ellos una concepción de la subalternidad como lugar de resistencia y sus teorías recibieron el aval de otras comunidades académicas.

En una etapa más avanzada de la discusión, apareció una nueva tradición: la pluralista liberal. Ésta sostenía que los medios son "independientes, tanto del Estado, como de los subsistemas sociales comprendidos dentro de los grupos políticos, económicos o de solidaridad" (Curran, 2005, p. 137), lo que les otorgaría "la libertad esencial que necesitan para responder a la totalidad de la sociedad” (Curran, 2005, p. 137). Rápidamente esta línea fue fuertemente cuestionada por evadir el hecho irrefutable de que los medios de comunicación pertenecen en su mayoría a los dueños de los medios de producción; a lo que los liberales contestaron con lo siguiente: 
El personal de los medios de comunicación cobró una mayor consciencia de su propio valor, adquirió mayor poder en la toma de decisiones personales como consecuencia de la creciente división del trabajo de las especializaciones dentro de las organizaciones mediáticas y desarrolló un compromiso con un conjunto de valores profesionales que trascendía las exigencias de los empresarios de los medios (Curran 2005, p. 138).

Como el tema es extenso y complejo, dejaremos el trazado histórico solo hasta este punto. Además es probable que ninguna de las tres perspectivas anteriormente mencionadas dé cuenta de la relación entre los medios y el poder, pues, como se verá a lo largo de este artículo, la discusión aún no está terminada. Una de las razones por las cuales este debate puede haberse debilitado es su delimitación disciplinar y por esto este artículo se ha propuesto insertar en él algunos de los postulados de la antropología, cuyo aporte puede darle nuevas luces a los estudios de comunicación en términos epistemológicos y metodológicos.

\section{Encuentros posibles entre la antropología y la comunicación en el estudio de los media}

En principio, si hablamos de medios, hablamos de cultura, y si hablamos de cultura, debemos acercarnos al debate antropológico que se ha planteado alrededor de este término. Como lo señala Thompson, "la comunicación mediática (...) se ocupa de la producción almacenamiento y circulación de materiales significativos para los individuos que los producen y los reciben” (Thompson, 1998, p. 25). Es importante pensar en los medios de comunicación, no como una gran tecnología (en el sentido foucaultiano del término) cognoscente y sujeta a una única racionalidad, sino-como su nombre lo indica- como medios que posibilitan la producción, circulación y consumo de significados que hacen parte de la cultura. En este sentido, no es posible concebir estos medios por fuera de sistemas axiológicos, simbólicos y discursivos previamente instituidos, así como tampoco es posible pensar a la cultura dentro de una economía política disociable de la producción mediática.

La primera de las consecuencias que traerá pensar en los medios en estrecha relación con la cultura, será pensarlos como fenómenos sociales contextualizados. Esto significa que la comunicación "siempre forma parte de contextos sociales estructurados" (Thompson 1998, p. 26). Esta característica despliega dos variables fundamentales para el análisis:

La primera es que, aunque resulte "tentador concentrarse en el contenido simbólico de los mensajes de los media"(Thompson 1998, p. 26) es imprescindible "tener en cuenta los propósitos y recursos de aquellos que los producen (...) (y) las maneras en que son utilizados y comprendidos por aquellos que los reciben" (Thompson 1998, p. 60); 
"así como el complejo orden de las condiciones sociales que subyacen a la producción y circulación de tales mensajes” (Thompson 1998, p. 27). Lo segundo que se debe comprender es que estos contenidos simbólicos, que son la esencia de los mensajes transmitidos mediáticamente, son también contextuales, y en este sentido, varían en función de la ubicación histórica, geográfica, económica y política de la producción; por lo tanto tienen una estrecha relación con la estructura social de la cual forman parte; relación que, como se verá en la segunda parte de este texto, no es unilateral sino dialéctica.

\section{La relación entre estructura social y cultura}

Para continuar es necesario traer a colación a Stuart Hall, uno de los principales referentes de los estudios culturales, quien además dedicó una gran parte de su vida a entender las relaciones posibles entre los medios, la hegemonía y la subalternidad. La tesis de Stuart Hall es que la cultura (en un sentido idealista), en tanto correlato discursivo de la estructura social, tiene la función de legitimar la desigualdad inherente a dicha estructura. La estructura de una sociedad, según afirma Stuart Hall:

Está siempre constituida por una serie de prácticas complejas; cada una tiene su propia especificidad, sus propios modos de articulación y mantiene un "desarrollo desigual” al de otras prácticas conexas.

Cualquier relación que esté dentro de esta complejidad estructurada tendrá su registro, sus "efectos", en todos los otros niveles de la totalidad: el económico, social, político e ideológico; ninguno puede ser reducido o destruido por otro (Hall, 2010, p. 232).

Una comprensión marxista de la cultura, sostendrá que el nivel ideológico está compuesto por "ideas, significados, conceptos, teorías, creencias, etc., los términos mediante los que los hombres “descifran el sentido" de su mundo" (Hall, 2010, p. 226). "Dar sentido, de este modo es, fundamentalmente, localizarse a uno mismo y a la experiencia y condiciones propias, en los discursos ideológicos ya objetivados" (Hall, 2010, p. 228).Los medios de comunicación cumplen un papel fundamental en este proceso, al detentar, no el monopolio, pero sí un lugar privilegiado en la actividad de "producir y difundir formas simbólicas de manera generalizada" (Thompson, 1998, p. 47). La primera función cultural de los medios es, como Hall lo afirma:

El suministro y construcción selectiva del conocimiento social, de la imaginería social por cuyo medio percibimos los "mundos", las "realidades vividas" de los otros y reconstruimos imaginariamente sus vidas y las nuestras en un "mundo global" inteligible, en una "totalidad vivida” (Hall, 2010, p. 243). 
Es en este sentido que los medios de comunicación constituyen un lugar fundamental para la comprensión de las sociedades, en la medida en que, al configurarse de y como representaciones culturales, son "ocasiones en las que nosotros -como elementos integrantes de una cultura o sociedad- nos reflejamos y definimos, escenificamos nuestros mitos colectivos e historias" (Mac Aloon 1984, p. 1, en Dickey, 2013, p. 2), así como "nuestras preocupaciones más dominantes" y "nuestros dilemas más profundos" (Curran, 2005, p. 135).Stuart Hall, define lo anterior en los siguientes términos:

Los acontecimientos por sí mismos no pueden significar: hay que hacerlos inteligibles; y el proceso de inteligibilidad social se compone precisamente de las prácticas que traducen los acontecimientos "reales" (...) a una forma simbólica. (...) Esta es la práctica social de la significación: la práctica a través de la cual se cumple el "trabajo" de la representación cultural e ideológica (Hall, 2010, p. 248).

La función ideológica de los medios será entonces hacer comprensible y aceptable la desigualdad estructural. Esta función corresponde a lo que el Stuart Hall ha llamado: "El efecto ideológico de los medios de comunicación”(Hall, 2010). Por lo tanto, cualquier proceso de comunicación es ideológico y sostiene una estrecha relación con la estructura social en la que tiene lugar, ya sea celebrándola, manteniéndola y/o escondiendo sus lógicas.

Sin embargo, como se ha mencionado previamente, éste no es el único tipo de relación que existe entre comunicación y cultura. Y es precisamente aquí donde yace el mayor aporte de la disciplina antropológica contemporánea, ya que mediante sus métodos y su epistemología se puede entender que, si bien las sociedades responden a estructuras previamente instituidas, los sujetos no son entes pasivos, sino agentes activos dentro de éstas. En otras palabras: ni la producción simbólica es monopolio de una clase dominante, que opera en función de una única racionalidad, que en este caso sería el capitalismo, ni el "proceso de recepción está exento de problemas" (Thompson, 1998, p. 45).Como afirma Thompson, la recepción no se trata de "un proceso carente de perspectiva crítica a través del cual los productos son absorbidos por los individuos, como la esponja absorbe el agua" (Thompson, 1998, p. 45).

\section{La recepción como proceso}

En este sentido, si bien es cierto que los medios de comunicación cumplen un papel central en el sostenimiento de la estructura, no puede ser vista su función por fuera de las relaciones antropológicas que tanto a su alrededor, como en su interior, se tejen. La antropología, especialmente la antropología de los medios, ha hecho una serie de hallazgos que complejizan el estudio de estos múltiples procesos: 


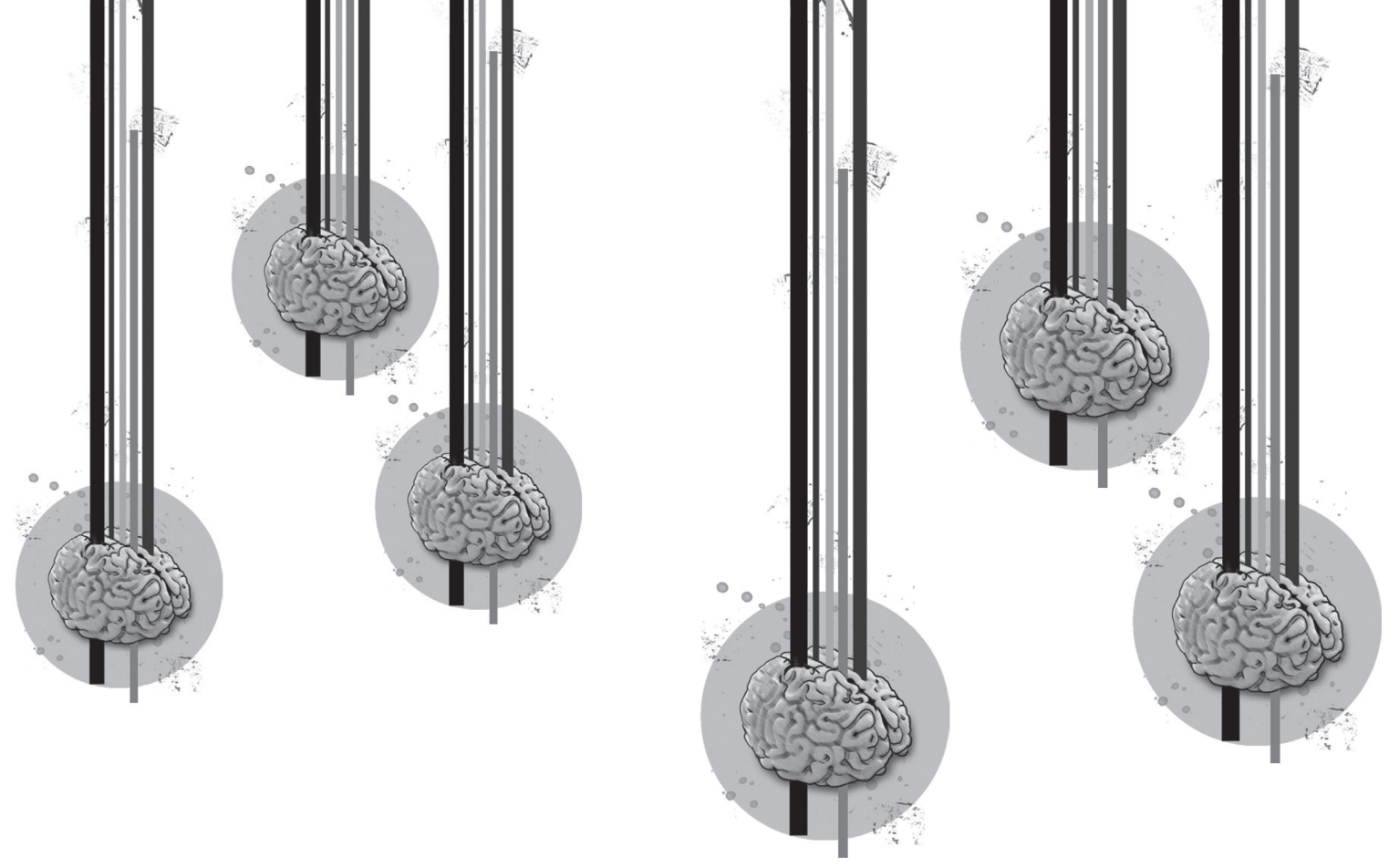

El primero tiene que ver con la recepción:

1. "La recepción de los productos mediáticos es una rutina, una actividad práctica que los individuos llevan a cabo como una parte integral de sus vidas cotidianas. Si queremos comprender la naturaleza de la recepción, entonces debemos desarrollar un tipo de aproximación que sea sensible a los aspectos rutinarios y prácticas de la actividad receptora" (Thompson, 1998, p. 61).

2. "Las maneras en que los individuos dan sentido a los productos mediáticos varía acorde con su bagaje social y sus circunstancias" (Thompson, 1998, p. 62). "Interpretamos los mensajes de los textos (...) a partir de las perspectivas de nuestras múltiples subjetividades, que han sido influidas por la "multitud de prácticas discursivas" (Mankekar 1993, p. 486, citada en Dickey, 2013, p. 5) con las que hemos estado en contacto a lo largo de nuestras vidas" (Dickey, 2013, p. 5).

3. "La recepción es una actividad situacional: los productos mediáticos son objeto de recepción por parte de individuos que siempre están ubicados en contextos socio-históricos específicos. Estos contextos están generalmente caracterizados por unas relaciones relativamente estables de poder y por un distinto acceso a los recursos acumulados de varios tipos" (Thompson, 1998, p. 63).

Estas aproximaciones, típicamente antropológicas, logran develar nuevas tensiones presentes en el proceso comunicacional: cada sujeto, contextualmente situado, fenomenológicamente afectado y estructuralmente ubicado, recepta los mensajes de manera diferenciada. Es decir: hay una incidencia, tanto del contexto sobre el sujeto, como del sujeto sobre el mensaje. Lo que nuevamente pone en crisis el carácter unilateral del proceso mediático. 


\section{La producción simbólica}

El segundo llamado que la disciplina antropológica hace al estudio de los medios es el de disociar la categoría monolítica del "productor”. Según afirma Sara Dickey:

Es menester llevar adelante esta tarea, porque la mayor parte de los medios de comunicación contemporáneos son producciones tan complejas que hasta las partes más accesibles de un proceso de creación exigen personal especializado, el cual ejerce sobre el producto final un control de índole variable tanto cualitativa como cuantitativamente (Dickey, 2013, p. 6).

Esta nueva variable del análisis mediático nos sugiere ponerle atención "al factor humano de los medios de comunicación y (...) (a) los amplios contextos en que éstos se hallan enraizados" (Dickey, 2013, p. 7). Ahora: si bien esta comprensión, más humana, del proceso mediático, matiza un poco la idea anterior de los medios como reflejo y producto de la ideología dominante, es necesario entender otra parte del proceso comunicativo que, tiene que ver con el contenido del mensaje y devela la compleja relación existente entre lenguaje e ideología.

Así como los procesos de recepción, los procesos de producción están enmarcados "por todo tipo de significados e ideas: el conocimiento operativo de las rutinas de producción, habilidades técnicas, ideologías profesionales, conocimiento institucional, definiciones y prejuicios, ideas preconcebidas sobre la audiencia, etc., enmarcan el tránsito del programa a través de esta estructura productiva” (Hall, 2004, p. 219).

John Thompson afirma que "la característica más destacada de la comunicación de masas es el hecho de que los productos estén disponibles, en principio, a una pluralidad de destinatarios" (Thompson, 1998, p. 44). Esto quiere decir que las representaciones, que estos medios ponen en circulación, son productos pensados bajo una lógica "industrial". Ésta es sin duda una aclaración importante, dado que plantea el hecho de que los códigos utilizados en la producción de mensajes deben tener un carácter extendido. Sin embargo, como lo dirá Hall: esto no quiere decir que su lectura no pueda ser resistente.

Stuart Hall, señala que "toda sociedad o cultura tiende a imponer a sus miembros sus propias segmentaciones, sus clasificaciones del mundo social, cultural y político (...). Éste se convierte en el orden cultural dominante, lo que no quiere decir unívoco o incuestionable" (Hall, 2004, p. 230). Para este autor, las representaciones mediáticas están conformadas por significados connotados que a su vez sostienen una "estrecha comunicación con la cultura, el conocimiento, la historia y con lo que el ha llamado "sentido común”, el cual está conformado por significados que han llegado a ser universalizados y naturalizados, (y) parecen las únicas formas disponibles de inteligibilidad (...) los únicos razonamientos universalmente válidos” (Marx, 1965, en Hall, 2010, p. 466). 
Otro de los elementos a tener en cuenta es que la legitimidad de estos significados está avalada por otras instituciones como el Estado, la familia, la iglesia, la escuela, entre otras. Esto es relevante pues, si bien es cierto la perpetuación del orden existente es una de las funciones fundamentales de la producción mediática, ésta no es su función por definición. Es decir, los medios requieren del consentimiento de la audiencia para tener su carácter masivo y es por ello que los medios emplean todo un repertorio de codificaciones hegemónicas. Pero, y en esto es bastante enfático Hall, no lo hacen únicamente "por su propio modo "desviado" de interpretar los acontecimientos, sino por la legitimidad de la gama o límites dentro de los cuales están funcionando sus codificaciones y decodificadores (Hall, 2010, p. 249)".

Para decirlo en otros términos: como la comunicación mediática, distinta a otros procesos sociales de interacción, no es un intercambio recíproco de ideas, gestos o representaciones. Sino que "los mensajes son producidos por un grupo de individuos y transmitidos a otros que están por lo general situados en emplazamientos espacial y temporalmente alejados del contexto de creación original" (Thompson, 1998, p. 45), una gran parte de los esfuerzos, las energías y la creatividad de las corporaciones televisivas y de otras empresas mediáticas comerciales van dirigidos e identificar el modo de agradar a la audiencia” (Curran, 2005, p. 139). Quienes forman y han formado parte de los medios de comunicación han desarrollado una variedad de técnicas para asegurar que sus mensajes tengan cierta efectividad en el momento de la decodificación, “desde la utilización de una fórmula bien escogida, con gancho y que atrae a una audiencia predecible (como las series televisivas y las películas divididas en partes) hasta la investigación de mercado y el control regular del tamaño y respuesta de la audiencia" (Thompson, 1998, p. 50).

\section{Sobre la etnografía de recepción}

En este texto pudimos ver algunas de las variables que influyen en la producción mediática y que la hacen cercana y aliada al poder. Sin embargo, también es posible ver cómo estas variables se retroalimentan. Dado que una de estas variables es la audiencia, y dado que la audiencia está conformada por agentes activos, pensantes y contextualmente situados, es importante devolverle el poder negociador y transformador a estos agentes. En este sentido la antropología tiene mucho que enseñarnos. Ya existe una línea investigativa en EE.UU. que propone nuevos marcos teóricos y metodológicos para reflexionar sobre la producción y recepción mediática. A continuación cito tres ejemplos que son pertinentes para complementar la discusión:

- El primero es "Interpretando la(s) cultura(s) después de la televisión: sobre el método” (2006), de la antropóloga Lila Abu-Lughod. En este texto, la autora intenta describir, a partir del estudio etnográfico, las maneras en las que los receptores interpretan los discursos emitidos por la televisión. Para ella, una investigación sobre audiencia debe basarse en la descripción de "un conjunto de prácticas y discursos 


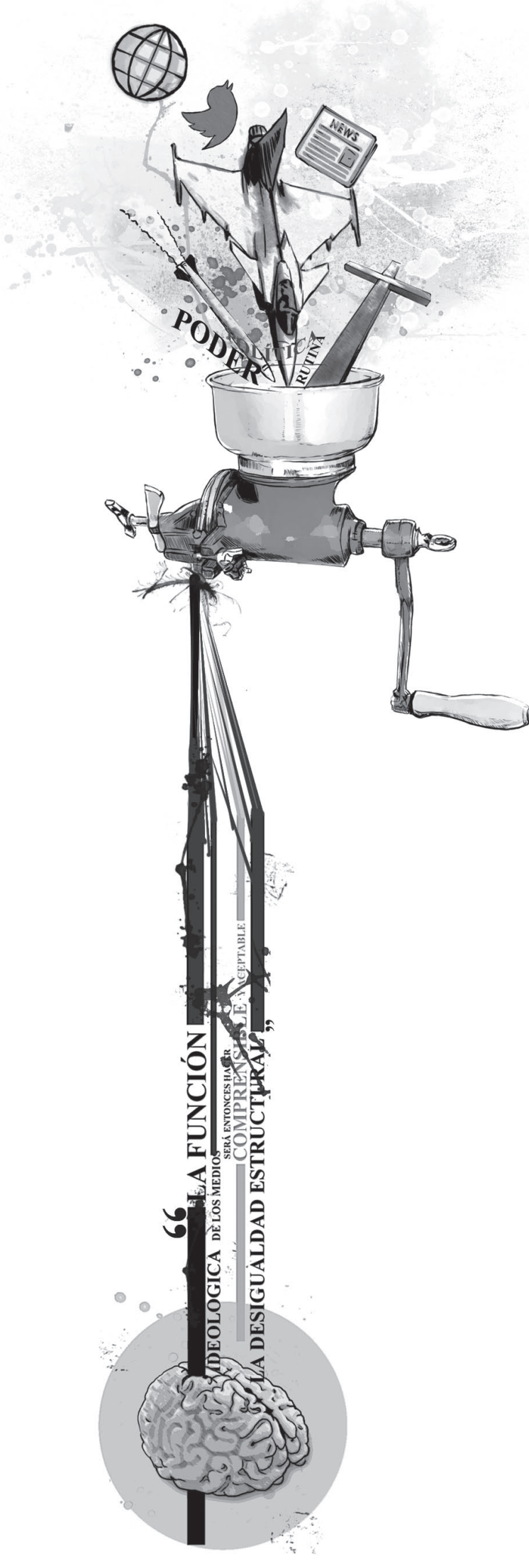

cotidianos dentro de los cuales se sitúa el complejo acto de ver televisión y a través de los cuales este mismo acto complejo se constituye" (Silverstone, 1994, citado en Lughod, 2006, p. 122). Es por esto que para ella, la etnografía, como método, es la única vía para dilucidar el hecho de que los mensajes transmitidos por los medios de comunicación se articulan con la concepción de las personas sobre sí mismas y su mundo (Abu-Lughod, 2006, p. 122).

- En esta misma vía, el estudio de Veena Das sobre el consumo de Soap Operas en la India, resalta el hecho de que la televisión, en tanto objeto doméstico, tiene una manera particular de consumirse, es decir, que al estar confinada al mundo del hogar, suele ser visto o por la familia o por miembros de ella de manera solitaria. Lo interesante de esta postura es que, para Das, las imágenes no mueren cuando son vistas, sino que se transforman en este entorno cerrado, convirtiéndose en objetos de conversaciones a partir de las cuales son reelaborados e interpretados. A esto le llama el autor: la vida social de las imágenes, y su estudio solo puede darse en contextos particulares, a partir de los cuales se puede dar cuenta de la complejidad de la relación entre lo que sucede en la pantalla y lo que sucede en la vida doméstica.

- Y el último ejemplo es el estudio de Purnima Mankekar, llamado "Television Tales and a Woman's Rage: A Nationalist Recasting of Draupadi's Disrobing" (1993), en el que investiga la recepción de poemas épicos entre jóvenes y mujeres en Delhi y Londres. Lo que intenta indagar la autora es ¿cómo espectadores en situaciones diferentes pueden llegar a interpretar también de manera diferenciada los mensajes? Es decir, a negociar activamente los significados proporcionados por los medios de comunicación (Mankekar, 1993, p. 479). Mankekar concluye en su investigación que los espectadores se apropian selectivamente de los relatos o incluso terminan refutándolos por completo. 
Estas tres investigaciones coinciden en su metodología: las tres son etnografías de consumo y hacen hincapié en el hecho de que "los públicos pueden interpretar el contenido de la televisión de una forma que dista mucho de los objetivos perseguidos por los creadores de programas" (Dickey, 1997). Éste es un enfoque bastante útil para antropólogos y comunicadores aunque, en términos operativos, puede llegar a ser bastante dispendioso.

\section{Nota de Autor. A modo de conclusión}

Para concluir, mi sugerencia es ver en los procesos mediáticos una dialéctica inherente. Por lo tanto, un estudio sobre medios, debe entonces dar cuenta de los siguientes aspectos, debidamente contextualizados:

- La audiencia, ya sea como agentes aislados, o como conjunto: En el primer caso será necesario, mediante métodos etnográficos, dar cuenta de cómo las personas interpretan los mensajes a partir de su propia experiencia y posición. En el segundo caso es menester comprender que, en tanto conjunto, la audiencia tiene el poder de cambiar por completo los contenidos aportados por los medios de comunicación, pues ellos dependen en gran medida de la aceptación del público.

- Los sistemas simbólicos: Los sistemas simbólicos hacen parte de sistemas culturales más amplios, pero a través de éstos es posible ver cómo son representadas distintas categorías sociales. Estos sistemas cambian en función de la ubicación histórica y geográfica pues en función de estas dos variables también cambian las estructuras de poder. Las mujeres, por ejemplo, no son hoy representadas como hace unas décadas, ni son representadas en Colombia de la misma manera que en la India, esto sucede porque pertenecemos a sistemas epistémicos y culturales distintos.

- Los medios como instituciones: Los medios de comunicación son aparatos social, económica y técnicamente organizados para la producción de mensajes y signos. En sociedades como la nuestra, estos medios pertenecen a oligopolios con pretensiones económicas y de poder específicas, que no se pueden eludir. Mapear estas alianzas entre mercado, política y medios también es necesario a la hora de intentar comprender las producciones mediáticas.

- Los productores o codificadores: Como lo afirma Sara Dickey, aún hay un camino por recorrer en la deconstrucción de la categoría del "productor", ya que ésta sigue siendo monolítica. Los equipos de producción suelen estar conformados por personas con diferencias de clase, ideología, etnia, región de procedencia, etc., lo que los hace distintos en su modo de interpretar el mundo. Aunque no es la vía más fácil, la etnografía puede ser un método útil a la hora de exponer las formas disimiles y complejas en que los productores operan. 


\section{Referencias}

Abu-Lughod, Lila. (2006). Interpretando la(s) cultura(s) después de la televisión: sobre el método, Iconos. Revista de Ciencias Sociales. No 24, Quito, enero 2006, pp. 119-141, FLACSO-Ecuador.

Curran, James (2005). Medios de comunicación y poder en una sociedad democrática, Hacer Editorial, Barcelona, pp. 121-171.

Debray, Régis (2001). Introducción a la mediología, Paidós, Barcelona, pp. 53-89.

Dickey, Sara (2013). "La antropología y sus contribuciones al estudio de los medios de comunicación”, Facultad de Ciencias Sociales, Universidad de Buenos Aires.

Hall, Stuart (2010). Sin garantías. Trayectorias y problemáticas en estudios culturales, Restrepo, Eduardo; Walsh, Catherine y Vich, Víctor (eds.), Instituto de Estudios Peruanos/Universidad Javeriana/ Universidad Andina Simón Bolívar, Quito, pp. 221-254.

Hall, Stuart (2004). "Codificación y descodificación en el discurso televisivo” en Cuadernos de Información y Comunicación, 9, Universidad Complutense de Madrid, pp. 210-236.

Mankekar, Purmina (1993) “Television Tales and a Woman's Rage: A Nationalist Recasting of Draupadi's Disrobing" en Public Culture N ${ }^{\circ}$ 3:469 492.

Thompson, John B (1998). Los medios y la modernidad, Paidós, Barcelona, pp. 25-68.

Williams, Raymond (1999). "Las comunicaciones como ciencia cultural", primera reimpresión, en Martín-Barbero, Jesús y Silva, Armando (Comp.), Proyectar la comunicación, Tercer Mundo Editores, Bogotá, pp. 70-81.

Recibido: octubre 30 / Aprobado: noviembre 20 de 2015

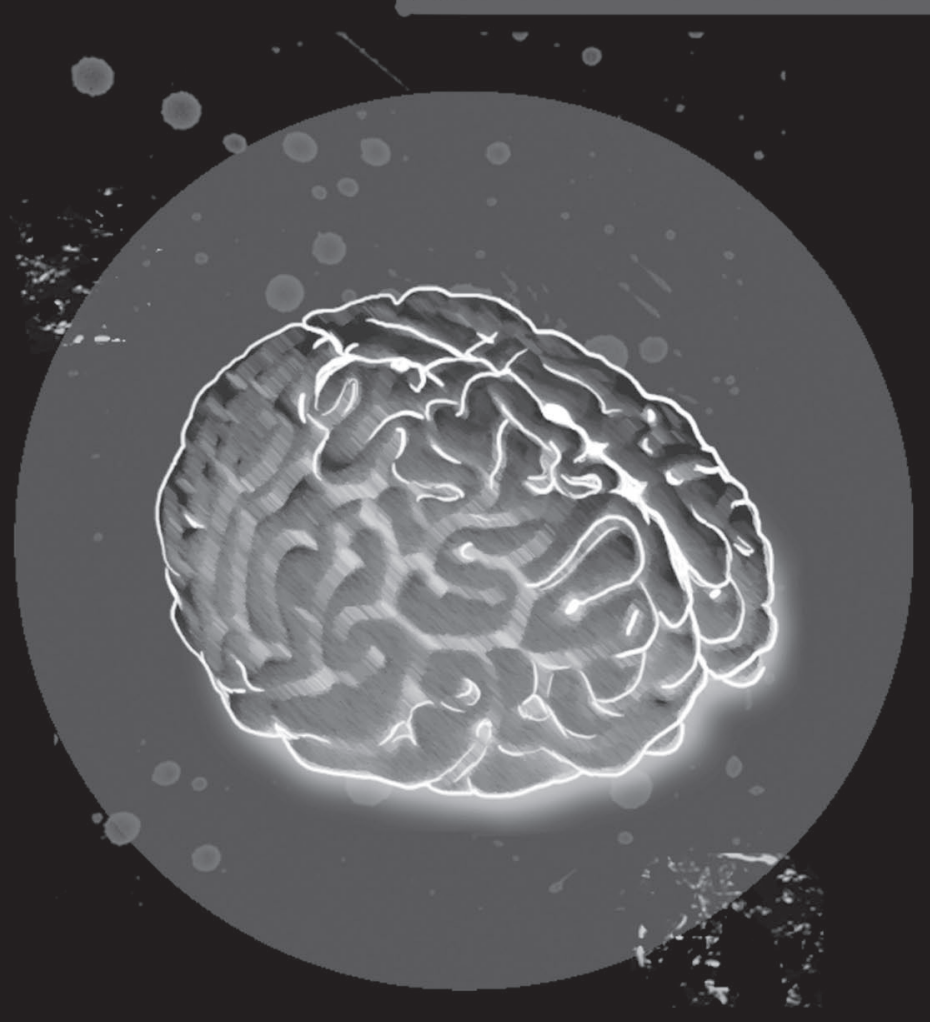

